\title{
Prognostic value of assessment of stool and serum IL-1 $\beta$, IL-1ra and IL-6 concentrations in children with active and inactive ulcerative colitis
}

\author{
Andrzej Wędrychowicz ${ }^{1}$, Przemysław Tomasik² ${ }^{2}$ Andrzej Zając ${ }^{3}$, Krzysztof Fyderek ${ }^{1}$
}

\author{
${ }^{1}$ Department of Pediatrics, Gastroenterology and Nutrition, Jagiellonian University \\ Medical College, Krakow, Poland \\ ${ }^{2}$ Department of Clinical Biochemistry, Jagiellonian University Medical College, Krakow, \\ Poland \\ ${ }^{3}$ Department of Pediatric Surgery, Jagiellonian University Medical College, Krakow, \\ Poland
}

Submitted: 20 February 2017

Accepted: 5 June 2017

Arch Med Sci 2018; 14, 1: 107-114

DOI: https://doi.org/10.5114/aoms.2017.68696

Copyright $\odot 2017$ Termedia \& Banach

\section{Abstract}

Introduction: Interleukin-1 $\beta$ (IL-1 $\beta$ ), interleukin-1 receptor antagonist (IL-1 ra) and interleukin-6 (IL-6) contribute to the pathogenesis of ulcerative colitis (UC). The aim of our study was to evaluate the serum and stool IL-1 $\beta$, IL-1ra and IL-6 concentrations as potential prognostic factors in children with UC. Material and methods: Thirty-eight children with UC (20 active, 18 inactive) and 14 healthy controls were prospectively included in the study. IL-1 $\beta$, IL-1ra and IL-6 concentrations were measured in serum and stool supernatants at inclusion to the study using ELISA immunoassays. The children were followed up over 5 years, and at each follow-up clinical disease activity, quantity and severity of relapses, nutritional status, endoscopic and histopathologic activity, disease complications and the treatment regimen were evaluated.

Results: In children with active and inactive UC who had relapsed during a 5 -year follow-up period compared to the non-relapse groups we found significantly increased serum IL-1 $\beta$ (1.34 vs. $0.98 \mathrm{pg} / \mathrm{ml}, p<0.05$, and 1.02 vs. $0.68 \mathrm{pg} / \mathrm{ml}, p<0.01$, respectively,) and IL-1ra (718.0 vs. $453.2 \mathrm{pg} / \mathrm{ml}$, $p<0.05$, and 567.4 vs. $365.1 \mathrm{pg} / \mathrm{ml}, p<0.01$, respectively). Additionally, in children who had experienced complications during a 5 -year follow-up period we observed significantly increased serum and stool IL-1 $\beta(p<0.05)$ and serum IL-1ra $(p<0.01)$ compared to the group without complications. Conclusions: We concluded that serum IL-1 $\beta$ and IL-1ra and to a lesser extend stool IL-1 $\beta$ concentrations may be useful prognostic factors in children with active and inactive UC over a short-term follow-up period, which may help to identify children that require more aggressive therapy due to an increased risk of relapse or complications resulting from UC.

Key words: interleukin, relapse, complication, ulcerative colitis, children.

\section{Introduction}

Inflammatory bowel diseases (IBD), including ulcerative colitis (UC) and Crohn's disease (CD), are chronic diseases of the gastrointestinal tract, characterized by relapsing intestinal inflammation. Both UC and CD share similar clinical symptoms including chronic diarrhea, abdominal pain, weight loss and growth failure [1].

\author{
Corresponding author: \\ Andrzej Wędrychowicz \\ MD, PhD \\ Department of Pediatrics, \\ Gastroenterology \\ and Nutrition \\ Jagiellonian University \\ Medical College \\ 265 Wielicka St \\ 30-663 Krakow, Poland \\ Phone: +48 126582011 \\ Fax: +48 126581088 \\ E-mail: andrzej. \\ wedrychowicz@uj.edu.pl
}


Although the exact cause of IBD is still unknown, there is evidence to support an essential role of the mucosal immune system in the initiation and perpetuation of inflammation [2]. Recent studies have identified bacterial microflora-derived antigens which adversely affect immunological reactions in the gut $[3,4]$.

Pro-inflammatory cytokines produced by the cells of the immune system, such as tumor necrosis factor (TNF)- $\alpha$, interleukin (IL)-1 $\beta$, IL-1 receptor antagonist (ra) and IL-6 play a major role in the pathogenesis of IBD [5].

Results from previous studies suggest that an imbalance of IL-1 $\beta$ and IL-1ra concentrations plays a crucial role in the early stages of IBD development. The elevated plasma and tissue IL-1ra concentrations observed in patients with IBD suggest that IL-1ra may be responsible for downregulating inflammatory pathways [6]. The IL-1ra/IL-1 ratio has been found to be negatively correlated with IBD activity, and remains constant in uninvolved areas of CD and in control patients [7]. The mucosal imbalance of intestinal IL-1 and IL-1 ra observed in IBD patients suggests that insufficient production of endogenous IL-1ra may contribute to the pathogenesis of chronic gut inflammation [8].

Elevated serum IL-1 $\beta$, IL-1ra and IL- 6 concentrations have been reported in patients with active UC and CD in multiple studies [9-11]. Similar observations have been made in studies which investigated the mucosal and fecal concentrations of IL-1 $\beta$, IL-1ra and IL- 6 in children and adults with active and inactive IBD [12-15].

Ulcerative colitis is a chronic disease characterized by relapses and complications, which alter the clinical course of the disease in addition to impairing the development of the child. Various factors may be responsible for UC relapse, including infections, diet and lack of patient compliance. However, in the majority of cases it is not possible to identify the specific factor that triggered the disease relapse, and therefore it is not possible to prevent the relapse. Before the clinical symptoms of relapse are visible in the patient, multiple immunological and inflammatory reactions probably take place, and tracking these changes could be used for clinical predictive purposes.

Ulcerative colitis can also be associated with different complications, during both short- and long-term patient follow-up periods, including hemorrhage, toxic megacolon, malnutrition and hepatobiliary and thromboembolic diseases. While treatment complications often develop early after disease onset, they can develop much later in some patients.

Nevertheless, there is a lack of knowledge regarding prognostic factors that could be used to predict relapses or complications in chronic relaps- ing diseases such as UC. This would allow for the optimization of treatment regimens and could be used to improve the clinical care of UC patients, in addition to their improving developmental outcomes and quality of life.

The aim of this study was to evaluate serum and stool IL-1 $\beta$, IL-1ra and IL-6 concentrations for their potential use in the prediction of relapses and complications resulting from UC in children.

\section{Material and methods}

Thirty-eight children with UC (20 active, at the time of onset of the disease, and 18 inactive, at the remission of the disease) and 14 healthy children (controls) were prospectively included in the study. The active UC group consisted of 10 boys and 10 girls with a mean age of 10.6 years (range: 2-15 years), and the inactive UC group consisted of 8 boys and 10 girls with a mean age of 11.7 years (range: 3.5-18 years). The active UC group consisted of newly diagnosed patients with clinical symptoms of UC, while the inactive UC group consisted of patients diagnosed previously, without any symptoms of UC at the time of inclusion. The mean time of UC duration in the inactive $U C$ group was 1.5 years (range: $0.5-2$ years). The control group consisted of 7 boys and 7 girls with a mean age of 11 years (range: 3.5-16 years), without any pathological gastrointestinal tract symptoms.

The diagnosis of UC was based on the Porto criteria [16]. The UC children were followed up over a 5-year period from their enrollment in the study. At each follow-up they were evaluated for clinical disease activity, number and severity of relapses, nutritional status, complications and treatment regimen.

The clinical activity of UC was assessed according to the modified Truelove-Witts clinical activity index, and clinical remission was defined as a total score of $<2$ points [17, 18].

As there is currently no established standard definition of UC relapse, we defined a clinical relapse of UC as the worsening of symptoms or the emergence of new symptoms that increased the modified Truelove-Witts clinical activity index score of $>2$ points, as well as if the treatment regimen required modification, which included increasing the doses of current drugs or introducing new ones.

During the follow-up period, relapse was observed in a total of 14 patients in the active UC group after a median 1.6 years of follow-up (range: 1.1-1.8 years) and 7 patients from the inactive UC group after a median 2.7 years of follow-up (range: 1.8-3.6 years of follow-up). Complications of UC were diagnosed in 5 children in the active UC group after a median 1.8 years of follow-up (range: $0.5-3.2$ years) (severe hemorrhage -2 patients, vein thrombosis - 1 patient, liver disease - 
2 patients) and 6 children in the inactive UC group after a median 2.6 years of follow-up (range: 1.64.5 years) (malnutrition -2 patients, osteoporosis - 2 patients, liver disease -2 patients) over the 5 -year follow-up period.

An improvement in nutritional status was defined as an increase in body weight, the Cole index and BMI z-score of the patient, when compared to their baseline values. The Cole index was defined as the weight/height-for-age ${ }^{2}$, and consisted of the squared weight/height ratio, expressed as the percentage of this ratio as calculated for a reference child of the same age and sex [19].

Control ileocolonoscopy was performed on every relapsed patient. Their endoscopic activity was assessed according to the Mayo UC endoscopic score index, and their histopathological activity was assessed according to the histopathological Gupta score index [20, 21].

The initial pharmacological treatment used for children in the active UC group consisted of 5-amino salicylates (sulphasalazine and mesalazine), antibiotics (metronidazole) and steroids (prednisone), depending on the activity of the disease. In the inactive UC group the pharmacological treatment included 5-amino salicylates (sulphasalazine and mesalazine) and thiopurines (azathioprine) as maintenance therapy [22-24].

IL-1 $\beta$, IL-1ra and IL- 6 concentrations were measured in serum and stool supernatants at inclusion in the study using commercial quantitative ELISA immunoassays (R\&D Systems, USA).

Stool supernatants were prepared from fresh stool samples (20-50 g), which were collected and weighed in sterile containers after the individual had defecated. The stool samples were then emulsified in an equal volume of phosphate buffered saline (PBS, pH 7.2) containing protease inhibitors (soy trypsin inhibitor and phenylmethylsulfonyl fluoride, $1 \mathrm{mg} / \mathrm{ml}$; Gibco, USA) and centrifuged at $10,000 \mathrm{rpm}$ for $20 \mathrm{~min}$. Afterwards, the supernatant was filtered through a $0.45 \mu \mathrm{m}$ micropore filter (Millipore, USA), collected and stored at $-70^{\circ} \mathrm{C}$ until the ELISA assays [25-27].

The study protocol was approved by the JagielIonian University Bioethical Committee. Informed consent was obtained from all patients enrolled in the study, either from the legal guardian of patients aged under 16 years or from the patients themselves if aged over 16 years.

\section{Statistical analysis}

Statistical analyses were performed using Statistica 10 software (StatSoft Inc., Tulsa, OK, USA) using the Mann-Whitney $U$ test, Wilcoxon signedrank test and Spearman's rank correlation test. All results were presented as the median values and ranges, and $p<0.05$ was considered to be statistically significant in all analyses.

\section{Results}

The serum and stool IL-1 $\beta$, IL-1ra and IL-6 concentrations were significantly higher and the IL-1 ra/IL-1 $\beta$ ratio was lower in the active UC group compared to the inactive UC and control groups $(p<0.05)$ at the beginning of the study. In the inactive UC group both the serum and stool sample IL-1 $\beta$, IL-1ra and IL-6 concentrations and IL-1ra/ $\mathrm{IL}-1 \beta$ ratios were comparable to controls (Table I).

Table I. Serum and stool IL-1 $\beta$, IL-1ra and IL-6 concentrations and IL-1ra/IL-1 $\beta$ ratio in active and inactive UC and control groups at the beginning of the study

\begin{tabular}{|c|c|c|c|}
\hline Parameter & Active UC group $(n=20)$ & Inactive UC group $(n=18)$ & Control group $(n=14)$ \\
\hline \multicolumn{4}{|c|}{$\mathrm{IL}-1 \beta[\mathrm{pg} / \mathrm{ml}]:$} \\
\hline Serum & $1.05(0.38-2.69)^{\star}$ & $0.87(0.39-1.9)$ & $0.33(0.11-1.0)$ \\
\hline Stool & $393.7(55.5-855)^{*}$ & $9.1(5.2-21.3)$ & $13.5(6.9-22.6)$ \\
\hline \multicolumn{4}{|c|}{ IL-1ra [pg/ml]: } \\
\hline Serum & $532.1(135.4-898.4)^{\star}$ & $418.1(205-946.9)$ & $322.5(183-737)$ \\
\hline Stool & $4950.3(910.7-6525)^{*}$ & $406.9(131.3-835)$ & $678.5(242-1570)$ \\
\hline \multicolumn{4}{|c|}{ IL-1ra/IL-1 $\beta$ ratio: } \\
\hline Serum & $450.4(267.4-1287)^{*}$ & $535.0(225-1108)$ & $856(367-2233)$ \\
\hline Stool & $10.8(6-32)^{\star}$ & $45.7(9.2-76.6)$ & $47.5(14-115)$ \\
\hline \multicolumn{4}{|c|}{ IL-6 [pg/ml]: } \\
\hline Serum & $5.8(1.3-10.7)^{\star}$ & $2.1(0.37-7.5)$ & $2.7(0.54-6)$ \\
\hline Stool & $13(4.6-31.8)^{\star}$ & $11.3(1.6-29.9)$ & $14.6(9.9-33)$ \\
\hline
\end{tabular}

Results are presented as median values and ranges. ${ }^{*} P<0.05$ when compared to inactive UC and control group. 
Table II. Serum IL-1 $\beta$, IL-1ra and IL- 6 concentrations and IL-1ra/IL-1 $\beta$ ratio in relapsing and non-relapsing patients during a 5 -year follow-up period for active UC group

\begin{tabular}{|c|c|c|c|}
\hline Parameter & Relapsing group $(n=14)$ & Non-relapsing group $(n=6)$ & $P$-value \\
\hline $\mathrm{IL}-1 \beta[\mathrm{pg} / \mathrm{ml}]$ & $1.34(1.04-2.69)$ & $0.98(0.38-1.36)$ & 0.05 \\
\hline IL-1ra [pg/ml] & $718.0(675.2-898.4)$ & $453.2(135.4-654.9)$ & 0.05 \\
\hline IL-1 ra/IL-1 $\beta$ ratio & $377.6(267.4-576.9)$ & $514.3(387.67-1287)$ & 0.05 \\
\hline IL-6 [pg/ml] & $7.48(3.50-10.7)$ & $4.74(1.3-7.47)$ & 0.23 \\
\hline
\end{tabular}

Results are presented as median values and ranges.

In the active UC group the serum IL-1 $\beta$ and IL-1ra concentrations were significantly higher and IL$1 \mathrm{ra} / \mathrm{IL}-1 \beta$ ratios were lower in patients who had relapsed during the 5-year follow-up period compared to patients who had not relapsed (Table II).

Serum IL-1 $\beta$ concentrations were also significantly higher in children who had relapsed in the active UC group and these patients had severe endoscopic activity $(n=9)$ compared to those with moderate and mild endoscopic activity $(n=11)$ as assessed at the disease onset $(p<0.05)$.

We observed higher serum and stool IL-1 $\beta$ ( $p<0.05$ for both sample types) and serum IL-1ra concentrations $(p<0.01)$ in patients in the active UC group who had experienced complications during the 5-year follow-up period compared to the group without any complications (Figure 1).

We did not find any statistically significant differences in IL-1 $\beta$, IL-1ra or IL-1ra\IL-1 $\beta$ ratio in stool between relapsing and non-relapsing UC patients.

For patients in the inactive UC group, we found that serum IL-1 $\beta$ and IL-1ra concentrations were

A

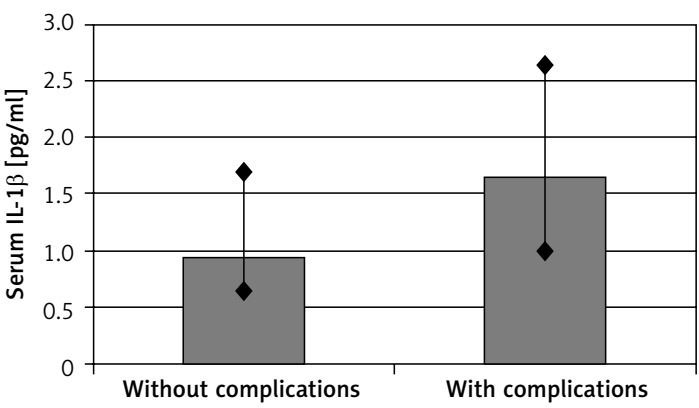

C

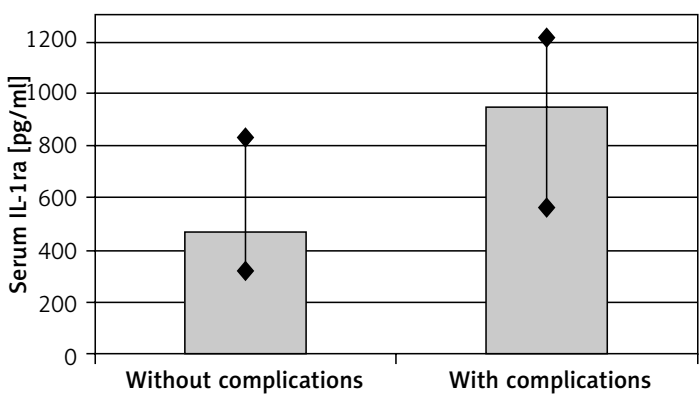

higher and the IL-1ra/IL-1 $\beta$ ratio was lower in patients who had relapsed during the 5-year follow-up period compared to the non-relapsing group (Table III).

Serum IL-1 $\beta$ and IL-1ra concentrations were higher ( $p<0.003$ and $p<0.005$, respectively) and the stool IL-6 concentration was lower $(p<0.05)$ in patients in the inactive UC group who had experienced complications within the 5-year follow-up period ( 6 children) compared to the group without complications.

We did not find any statistically significant differences or correlations between relapsing and non-relapsing UC patients regarding the clinical, endoscopic and histopathological activity of UC, disease location, disease duration, nutritional status or used treatment (Tables IV, V).

There were no statistically significant differences or correlations in IL-1 $\beta$, IL-1ra and IL- 6 concentrations and the IL- $1 \mathrm{ra} / \mathrm{IL}-1 \beta$ ratio in the active and inactive UC groups for clinical and histopathological activity scoring indexes, nutritional status, number and severity of relapses, compli-

\section{B}

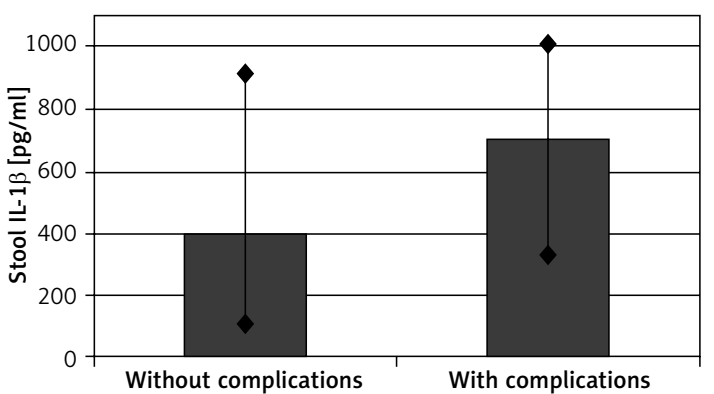

Figure 1. Concentrations of IL-1 $\beta$ in serum (A), stool (B) and IL-1ra in serum (C) in active ulcerative colitis (UC) patients who had experienced complications during the 5-year follow-up period compared to the group without any complications. Data are expressed as median values and ranges. Differences statistically significant with $p$-value $<0.05$ and $<0.01$, respectively 
Table III. Serum IL-1 $\beta$, IL-1 ra and IL- 6 concentrations and IL-1ra/IL-1 $\beta$ ratio in relapsing and non-relapsing patients during a 5-year follow-up period for inactive UC group

\begin{tabular}{|lccc|}
\hline Parameter & Relapsing group $(n=7)$ & Non-relapsing group $(n=11)$ & $P$-value \\
\hline $\mathrm{IL}-1 \beta[\mathrm{pg} / \mathrm{ml}]$ & $1.02(0.43-1.90)$ & $0.68(0.39-1.02)$ & 0.01 \\
\hline $\mathrm{IL}-1 \mathrm{ra}[\mathrm{pg} / \mathrm{ml}]$ & $567.4(358.3-946.9)$ & $365.1(205-520.9)$ & 0.01 \\
\hline $\mathrm{IL}-1 \mathrm{ra} / \mathrm{IL}-1 \beta$ ratio & $506.3(225-840.9)$ & $684.0(261.7-1108)$ & 0.05 \\
\hline $\mathrm{IL}-6[\mathrm{pg} / \mathrm{ml}]$ & $2.57(1.98-7.5)$ & $1.89(0.37-3.87)$ & 0.18 \\
\hline
\end{tabular}

Results are presented as median values and ranges.

Table IV. Differences between relapsing and non-relapsing patients of active UC group regarding activity of the disease and used treatment

\begin{tabular}{|lccc|}
\hline Parameter & Relapsing group $(n=14)$ & Non-relapsing group $(n=6)$ & $P$-value \\
\hline Clinical activity (Truelove-Witts index) & $5(3-10)$ & $4(3-9)$ & 0.79 \\
\hline Endoscopic activity (Mayo score) & $2(1-3)$ & $2(1-2)$ & 0.48 \\
\hline Histopathological activity (Gupta score) & $2(1-3)$ & $2(1-2)$ & 0.54 \\
\hline \begin{tabular}{l} 
Disease location: \\
\hline Left-sided
\end{tabular} & $15 \%$ & $16 \%$ & 0.68 \\
\hline Pancolitis & $85 \%$ & $84 \%$ & 0.74 \\
\hline Disease duration [weeks] & $6(3-16)$ & $5(2.5-20)$ & 0.86 \\
\hline \begin{tabular}{l} 
Nutritional status: \\
\hline BMI z-score
\end{tabular} & $-0.3(-0.7-0.3)$ & $-0.5(-0.7-0.1)$ & 0.37 \\
\hline Cole index & $85 \%(80-92 \%)$ & $83 \%(78-88 \%)$ & 0.28 \\
\hline Treatment: & $30 \%$ & $32 \%$ & \\
\hline \begin{tabular}{l}
$5-\mathrm{ASA}$ \\
\hline 5-ASA and antibiotics
\end{tabular} & $15 \%$ & $16 \%$ & 0.82 \\
\hline 5-ASA and antibiotics and steroids & $55 \%$ & $52 \%$ & 0.88 \\
\hline
\end{tabular}

Data are presented as median values and ranges. BMI - body mass index, 5-ASA - 5 aminosalicylic acid.

Table V. Differences between relapsing and non-relapsing patients of inactive UC group regarding activity of the disease and used treatment

\begin{tabular}{|c|c|c|c|}
\hline Parameter & Relapsing group $(n=7)$ & Non-relapsing group $(n=11)$ & $P$-value \\
\hline Clinical activity (Truelove-Witts index) & $1(0-3)$ & $1(0-2)$ & 0.83 \\
\hline Endoscopic activity (Mayo score) & $2(1-3)$ & $1(1-2)$ & 0.36 \\
\hline Histopathological activity (Gupta score) & $2(1-3)$ & $1(1-2)$ & 0.45 \\
\hline \multicolumn{4}{|l|}{ Disease location: } \\
\hline Left-sided & $15 \%$ & $9 \%$ & 0.43 \\
\hline Pancolitis & $85 \%$ & $91 \%$ & 0.78 \\
\hline Disease duration [years] & $1.3(0.5-2)$ & $1.6(0.8-2)$ & 0.82 \\
\hline \multicolumn{4}{|l|}{ Nutritional status: } \\
\hline BMI z-score & $-0.2(-0.6-0.4)$ & $-0.4(-0.7-0.2)$ & 0.52 \\
\hline Cole index & $87 \%(82-98 \%)$ & $85 \%(81-93 \%)$ & 0.58 \\
\hline \multicolumn{4}{|l|}{ Treatment: } \\
\hline 5-ASA & $15 \%$ & $30 \%$ & 0.73 \\
\hline 5-ASA and thiopurines & $85 \%$ & $70 \%$ & 0.81 \\
\hline
\end{tabular}

Data are presented as median values and ranges. BMI - body mass index, 5-ASA - 5 aminosalicylic acid. 
cations and treatment regimen during the 5-year follow-up period.

\section{Discussion}

Ulcerative colitis is a chronic intestinal disorder characterized by relapses with exacerbation of intestinal symptoms. However, despite appropriate maintenance therapy, it is impossible to predict and prevent flare-ups in clinical practice.

The prevention and early treatment of relapses are essential in the medical care of UC patients. This could potentially reduce the number and severity of relapses, increase the quality of life of UC patients and reduce the financial costs associated with treatment.

Numerous previous studies have aimed to determine the prognostic factors associated with UC relapses, including analyzing acute phase proteins, fecal occult blood tests or stool calprotectin concentration.

Kuriyama et al. examined the feasibility of predicting UC relapses using the quantitative immunochemical fecal occult blood test in adult patients with inactive UC over a 20-month follow-up period. They observed an increase in fecal hemoglobin concentration in $20 \%$ of patients 2 to 4 weeks before any clinical symptoms of relapse [28]. The fecal occult blood test is cheap and easy to perform, but it is not specific for UC, and positive results could be associated with infections, menstruation or colorectal cancer.

Fecal calprotectin is the most frequently used predictive factor for IBD in clinical practice due to its high correlation with endoscopic and histopathologic activity $[29,30]$. Tibble et al. found that elevated concentrations of fecal calprotectin in patients with a quiescent course of IBD had 90\% sensitivity and $83 \%$ specificity for predicting relapse [31]. A study by Yamamoto et al. evaluated the predictive ability of a single point fecal calprotectin and lactoferrin assessment for relapse in adult patients with inactive UC. They followed up a group of UC patients for 12 months and found that increased concentrations of both fecal calprotectin and lactoferrin were associated with clinical relapse of the disease [32]. However, fecal calprotectin was not found to be correlated with IBD activity in patients after surgical resections, while fecal lactoferrin was higher in patients with UC compared to those with $C D$, and was considered to be a superior marker for UC [29].

Oruç et al. investigated a group of 45 newly diagnosed IBD patients, and compared the diagnostic value of assessment of serum acute phase reactant proteins, including C-reactive protein, procalcitonin and erythrocyte sedimentation rate (ESR). They found that C-reactive protein was the most sensitive and useful marker of IBD activity when compared to other acute phase reactants. The authors of the study suggested that procalcitonin and ESR have no clinical value in the assessment of disease activity in IBD [33].

In our study, we focused on cytokines as immunological mediators as they play a key role in determining the nature of immunomodulation during inflammatory responses and may be helpful in clinical assessment in different immune-related diseases [5, 34]. Cytokines are related to the synthesis and secretion of immunological mediators such as leukotrienes, prostaglandins and reactive oxygen metabolites, and these are associated with the severity of inflammatory reactions.

Nevertheless, cytokines may also be involved in immunological reactions resulting in perpetuation of inflammation in the colon leading to relapses and complications. Long-lasting stimulation and continuous production of immunological mediators (e.g. ILS, TNF) may result in involvement of another organ and extraintestinal manifestations (skin, joint or liver diseases) or impaired metabolic reactions (osteoporosis, malnutrition).

Therefore, we speculated that altered concentrations of these cytokines may affect disease activity [35].

We used a similar method to previous studies for the single point stool assessment of the immunological mediators IL-1 $\beta$, IL- 6 and IL-1ra, which have been confirmed to contribute to the pathogenesis of UC. For a more sensitive assessment we assessed the IL concentrations in both serum and stool samples. The serum sample was used to assess the general immunological stimulation in the peripheral circulation, whereas the stool sample was used to assess the local immunological stimulation, close to the inflamed intestine.

In our study, we found a higher serum IL-1ra concentration and a lower IL-1ra/IL-1 $\beta$ ratio in children with active UC who had relapsed, and higher serum and stool IL-1 $\beta$ and serum IL-1ra concentrations in children with active UC who had experienced complications over the 5-year follow-up period, compared to the group without complications. We also found that serum IL-1 $\beta$ and IL-1ra concentrations were higher in the inactive UC group with relapses during the 5-year follow-up period compared to the group that had not-relapsed.

However, in our study we did not analyze factors that potentially may provoke or influence the relapse in the course of the disease. During statistical analysis we did not find any statistically significant differences or correlations between relapsing and non-relapsing UC patients regarding the clinical, endoscopic and histopathological activity of UC, disease location, disease duration, nutritional status or used treatment. 
To date, there have been only a few studies that have assessed the clinical and prognostic value of immunological mediators including ILS.

Däbritz et al. studied 147 adults and 34 children with CD and UC to investigate the use of a novel marker of intestinal inflammation, neutrophil-derived protein S100A12. During a 3-year follow-up period they collected serum and stool samples from the patients for the assessment of this marker. The authors found that the concentrations of the S100A12 protein in the serum and fecal samples were correlated with other markers, disease activity and location of inflammatory lesions. Increased S100A12 levels were also associated with an increased relapse risk with high sensitivity and specificity. Däbritz et al. suggested that this protein could be used as a marker to predict relapse in IBD patients [36].

In another study by Däbritz et al. they assessed the use of serum granulocyte macrophage colony-stimulating factor (GM-CSF) autoantibodies as a disease relapse marker in IBD. They found that the concentration of serum GM-CSF autoantibodies was correlated with disease activity and location, and that increased serum levels could predict a clinical relapse of up to 6 months [37].

A study by Mahida et al. assessed the serum IL- 6 concentrations in adult patients with UC or CD. They found elevated IL-6 concentrations in the CD group but not in the UC group, in which IL-6 concentrations were comparable to controls. IL-6 concentrations were correlated with activity markers, especially in the CD group. However, the patients were not followed up, and therefore the relationship of IL- 6 concentrations with relapse and complications of IBD was not assessed [38].

A prospective study by Garcia-Sanchez et al. on 135 adult patients with IBD in remission evaluated the use of systemic cytokines including TNF- $\alpha$, IL- $1 \beta$ and IL- 6 as predictors of IBD relapse. The patients were followed up after a year, and $30 \%$ of patients were found to have relapsed. The authors did not find any association between cytokine concentration and risk of relapse and therefore suggested that the serum cytokine assessment had no clinical value in the prediction of IBD relapse [39]. In contrast to the results from Garcia-Sanchez et al., we found statistically significant differences between relapsing and non-relapsing patients. The differences between studies may be explained by the different patient population (adults vs. children) and the follow-up period (1 year vs. 5 years). Additionally, in our study we assessed the concentration of these cytokines in stool samples, which provided more information and increased the value of the study.

To our knowledge, no previous studies have assessed the predictive value of the concentra- tion of cytokines in stool samples of patients with IBD. Therefore, our study is the first to assess the value of stool ILs as predictors of relapse and complications in patients with IBD. Our results suggest that the clinical determination of stool and serum IL concentrations have a potential use as a non-invasive method of stratifying the risk of relapse and complications among children with UC.

In conclusion, clinical use of known biomarkers in predicting disease progression and in determination of the most efficient therapeutic strategies is limited [40]. None of them is sensitive or specific enough, which makes it difficult to predict the future disease progression of IBD [41]. Due to the confirmed immunological background of IBD, we hypothesized that immunological mediators, such as IL-1 $\beta$ and IL-1ra, could potentially be used for the assessment of disease activity in IBD.

The results of this study suggest that serum and to a lesser extend stool IL-1 $\beta$ and IL-1ra concentrations may be useful prognostic factors in children with active and inactive UC over a shortterm follow-up period. This could help to identify children who require more aggressive therapy due to an increased risk of relapse or complications associated with UC.

\section{Conflict of interest}

The authors declare no conflict of interest.

\section{References}

1. Hanauer SB. Inflammatory bowel disease: epidemiology, pathogenesis, and therapeutic opportunities. Inflamm Bowel Dis 2006; 12 Suppl. 1: S3-9

2. Wallace KL, Zheng LB, Kanazawa Y, Shih DQ. Immunopathology of inflammatory bowel disease. World J Gastroenterol 2014; 20: 6-21.

3. Abraham C, Medzhitov R. Interactions between the host innate immune system and microbes in inflammatory bowel disease. Gastroenterology 2011; 140: 1729-37.

4. Fyderek K, Strus M, Kowalska-Duplaga K, et al. Mucosal bacterial microflora and mucus layer thickness in adolescents with inflammatory bowel disease. World J Gastroenterol 2009; 15: 5287-94.

5. Neurath MF. Cytokines in inflammatory bowel disease. Nat Rev Immunol 2014; 14: 329-42.

6. Casini-Raggi V, Kam L, Chong YJ, Fiocchi C, Pizarro TT, Cominelli F. Mucosal imbalance of IL-1 and IL-1 receptor antagonist in inflammatory bowel disease. A novel mechanism of chronic intestinal inflammation. J Immunol 1995; 154: 2434-40.

7. Dionne S, D'Agata ID, Hiscott J, et al. Colonic explant production of IL-1 and its receptor antagonist is imbalanced in inflammatory bowel disease (IBD). Clin Exp Immunol 1998; 112: 435-42.

8. Cominelli F, Pizarro TT. Interleukin-1 and interleukin-1 receptor antagonist in inflammatory bowel disease. Aliment Pharmacol Ther 1996; 10 (Suppl. 2): 49-53.

9. Ligumsky M, Simon PL, Karmeli F, Rachmilewitz D. Role of interleukin 1 in inflammatory bowel disease-en- 
hanced production during active disease. Gut 1990; 31 : 686-9.

10. Wedrychowicz A, Stopyrowa J, Miezynski W, Fyderek K. Serum interleukin 1 beta and interleukin 1 receptor antagonist in children with active ulcerative colitis. Pediatr Pol 2004; 79: 971-6.

11. Hyams JS, Fitzgerald JE, Wyzga N, Treem WR, Justinich CJ, Kreutzer DL. Characterization of circulating interleukin-1 receptor antagonist expression in children with inflammatory bowel disease. Dig Dis Sci 1994; 39: 1893-9.

12. Raab Y, Hällgren R, Gerdin B. Enhanced intestinal synthesis of interleukin- 6 is related to the disease severity and activity in ulcerative colitis. Digestion 1994; 55: 44-9.

13. Ludwiczek O, Vannier E, Borggraefe I, et al. Imbalance between interleukin-1 agonists and antagonists: relationship to severity of inflammatory bowel disease. Clin Exp Immunol 2004; 138: 323-9.

14. Peterson CG, Sangfelt P, Wagner $M$, et al. Fecal levels of leukocyte markers reflect disease activity in patients with ulcerative colitis. Scand J Clin Lab Invest 2007; 67: 810-20.

15. Wedrychowicz A, Stopyrowa J, Fyderek K, Miezynski W. Stool interleukin 1 beta and interleukin 1 receptor antagonist concentrations in children with active ulcerative colitis and during recovery. Eur J Pediatr 2003, 162: 541-2.

16. IBD Working Group of the ESPGHAN. Inflammatory bowel disease in children and adolescents: recommendations for diagnosis - the Porto criteria. J Pediatr Gastroenterol Nutr 2005; 41: 1-7.

17. Vilela EG, Torres HO, Martins FP, Ferrari Mde L, Andrade MM, Cunha AS. Evaluation of inflammatory activity in Crohn's disease and ulcerative colitis. World J Gastroenterol 2012; 18: 872-81.

18. Ryzko J, Socha J, Woynarowski M, et al. Validation of disease activity score indexes for inflammatory bowel disease. Surg Childh Intern 1996; 1: 17-21.

19. Cole TJ, Donnet ML, Stanfield JP. Weight-for-height indices to assess nutritional status - a new index on a slide rule. Am J Clin Nutr 1981; 31: 1935-43.

20. Tontini GE, Bisschops R, Neumann H. Endoscopic scoring systems for inflammatory bowel disease: pros and cons. Expert Rev Gastroenterol Hepatol 2014; 8: 543-54.

21. Marchal Bressenot A, Riddell RH, Boulagnon-Rombi C, et al. The histological assessment of disease activity in ulcerative colitis. Aliment Pharmacol Ther 2015; 42: 957-67.

22. Panaccione R, Rutgeerts P, Sandborn WJ, Feagan B, Schreiber S, Ghosh S. Treatment algorithms to maximize remission and minimize corticosteroid dependence in patients with inflammatory bowel disease. Aliment Pharmacol Ther 2008; 28: 674-88.

23. Ordás I, Eckmann L, Talamini M, Baumgart DC, Sandborn WJ. Ulcerative colitis. Lancet 2012; 380: 1606-19.

24. Zhang Z, Kennedy H. Ulcerative colitis: current medical therapy and strategies for improving medication adherence. Eur J Gastroenterol Hepatol 2009; 21: 1-8.

25. Nicholls S, Stephens S, Braegger CP, Walker-Smith JA, MacDonald TT. Cytokines in stools of children with inflammatory bowel disease or infective diarrhea. J Clin Pathol 1993; 46: 757-60.

26. Wedrychowicz A, Stopyrowa J, Fyderek K, Miezynski W. Serum and stool interleukin 6 in active and inactive ulcerative colitis in children. Pediatr Wspolcz 2000; 3: $165-9$.
27. Saiki T, Mitsuyama K, Toyonaga A, Ishida H, Tanikawa K. Detection of pro- and anti-inflammatory cytokines in stools of patients with inflammatory bowel disease. Scand J Gastroenterol 1998; 33: 616-22.

28. Kuriyama M, Kato J, Takemoto K, Hiraoka S, Okada H, Yamamoto K. Prediction of flare-ups of ulcerative colitis using quantitative immunochemical fecal occult blood test. World J Gastroenterol 2010; 16: 1110-4.

29. Abraham BP, Thirumurthi S. Clinical significance of inflammatory markers. Curr Gastroenterol Rep 2009; 11: 360-7.

30. Stawczyk-Eder K, Eder P, Lykowska-Szuber L, et al. Is faecal calprotectin equally useful in all Crohn's disease locations? A prospective, comparative study. Arch Med Sci 2015; 11: 353-61.

31. Tibble JA, Sigthorsson G, Bridger S, Fagerhol MK, Bjarnason I. Surrogate markers of intestinal inflammation are predictive of relapse in patients with inflammatory bowel disease. Gastroenterology 2000; 119: 15-22.

32. Yamamoto T, Shiraki M, Bamba T, Umegae S, Matsumoto K. Fecal calprotectin and lactoferrin as predictors of relapse in patients with quiescent ulcerative colitis during maintenance therapy. Int J Colorectal Dis 2014; 29: 485-91.

33. Oruç N, Ozütemiz O, Osmanoğlu N, Ilter T. Diagnostic value of serum procalcitonin in determining the activity of inflammatory bowel disease. Turk J Gastroenterol 2009, 20: 9-12.

34. Majak P, Jerzyńska J, Bojo M, et al. Cytokine profiling in exhaled breath condensate after exercise challenge in asthmatic children with post-exercise symptoms. Arch Med Sci 2016; 12: 778-84.

35. Neuman MG. Immune dysfunction in inflammatory bowel disease. Transl Res 2007; 149: 173-86.

36. Däbritz J, Langhorst J, Lügering A, et al. Improving relapse prediction in inflammatory bowel disease by neutrophil-derived S100A12. Inflamm Bowel Dis 2013; 19: 1130-8.

37. Däbritz J, Bonkowski E, Chalk C, et al. Granulocyte macrophage colony-stimulating factor auto-antibodies and disease relapse in inflammatory bowel disease. Am J Gastroenterol 2013; 108: 1901-10.

38. Mahida YR, Kurlac L, Gallagher A, Hawkey CJ. High circulating concentrations of interleukin-6 in active Crohn's disease but not ulcerative colitis. Gut 1991; 32: 1531-4.

39. García-Sánchez V, González R, Iglesias-Flores E, et al. Can systemic cytokines predict relapse of inflammatory bowel disease? Hepatogastroenterology 2010; 57 : 524-30.

40. Liverani E, Scaioli E, Digby RJ, Bellanova M, Belluzzi A. How to predict clinical relapse in inflammatory bowel disease patients. World J Gastroenterol 2016; 22: 1017-33.

41. Benitez JM, Louis E. Can we predict the high-risk patient? Dig Dis 2014; 32: 328-36. 